\title{
Comparison of underwater and conventional endoscopic mucosal resection for removing sessile colorectal polyps: a propensity-score matched cohort study
}

\section{(ㄷ)(i)}

\author{
Authors \\ Hsu-Chih Chien ${ }^{1,}{ }^{*}$, Noriya Uedo², Ping-Hsin Hsieh ${ }^{3,4, *}$ \\ Institutions \\ 1 Division of Epidemiology, Department of Internal \\ Medicine, University of Utah, Salt Lake City, Utah, United \\ States \\ 2 Department of Gastrointestinal Oncology, Osaka \\ International Cancer Institute, Osaka, Japan \\ 3 Department of Gastroenterology, Chimei Medical \\ Center, Tainan, Taiwan. \\ 4 Department of Gastroenterology, Fujen Catholic \\ University Hospital, New Taipei City, Taiwan
}

submitted 22.2.2019

accepted after revision 28.8.2019

\author{
Bibliography \\ DOI https://doi.org/10.1055/a-1007-1578 | \\ Endoscopy International Open 2019; 07: E1528-E1536 \\ (c) Georg Thieme Verlag KG Stuttgart · New York \\ elSSN 2196-9736
}

\section{Corresponding author}

Ping-Hsin Hsieh, MD, Chimei Medical Center, Tainan, Taiwan, Department of Gastroenterology, No.901, Zhonghua Rd., Yongkang District, Tainan City Tainan 71004, Taiwan

Fax: +886-06-281-2811

b86401099@gmail.com

\# Supplementary material

Online content viewable at:

https://doi.org/10.1055/a-1007-1578

\section{ABSTRACT}

Background and study aims Endoscopic mucosal resection (EMR) is a standard method for removing sessile colorectal polyps $\geq 10 \mathrm{~mm}$. Recently, underwater EMR (UEMR) has been introduced as a potential alternative. However, the effectiveness and safety of UEMR compared with conventional EMR is un clear.

Patients and methods In this 1:1 propensity score (PS) matched retrospective cohort study, we compared the en bloc resection rates, procedure time, intraprocedural and delayed bleeding rates, and incidence of muscle layer injury. We also performed subgroup analyses by sizes of polyps ( $<20 \mathrm{~mm}$ and $\geq 20 \mathrm{~mm}$ ).

Results Among 350 polyps in 315 patients from August 2012 to November 2017, we identified 121 PS-matched pairs. Mean polyp size was $16.8 \mathrm{~mm}$. With similar en bloc resection rates (EMR: $82.6 \%$ vs. UEMR: $87.6 \%$, rate difference: $5.0,95 \%$ confidence interval [ $95 \% \mathrm{CI}]:-4$ to $13.9 \%)$, UEMR demonstrated a shorter resection time $(10.8 \mathrm{~min}$ vs. 8.6 min, difference: $-2.2 \mathrm{~min}, 95 \% \mathrm{Cl}:-4.1$ to $-0.3 \mathrm{~min}$ ) and a lower intraprocedural bleeding rate $(15.7 \%$ vs. $5.8 \%$, rate difference: $-9.9 \%, 95 \% \mathrm{Cl}:-17.6$ to $-2.2 \%)$. Incidence of delayed bleeding and muscle layer injury were low in both groups. For polyps $<20 \mathrm{~mm}$, effectiveness and safety outcomes were similar in both groups. For polyps $\geq 20 \mathrm{~mm}$ (42 PS-matched pairs), the UEMR group has a comparable en bloc resection rate with shorter procedure time and superior safety outcomes

Conclusions UEMR achieved an en bloc resection rate comparable to conventional EMR with less intraprocedural bleeding and a shorter procedure time.

\section{Introduction}

Colorectal cancer (CRC) is the third most common cause of cancer mortality worldwide [1]. Colonoscopic removal of adenomatous polyps has been reported to reduce CRC-related mortality by $53 \%$ [2]. Endoscopic mucosal resection (EMR) is a standard method for removing sessile colorectal polyps larger

\footnotetext{
* Drs. Chien and Hsieh: These authors contributed equally.
}

than $10 \mathrm{~mm}$ [3 - 5]. EMR involves submucosal injection of fluid underneath a polyp to decrease risks of accidentally ensnaring the muscularis propria and causing a transmural thermal injury that can lead to perforation of the colon $[6,7]$.

Recurrence after EMR is not uncommon and could be problematic. Four known factors are associated with post-EMR recurrence. First and most important is piecemeal resection, i.e. en bloc resection failed or was impossible. Local recurrence occurs after $3 \%$ of en bloc EMRs and after $20 \%$ of piecemeal EMRs 
[8]. For piecemeal EMRs, three other factors associated with local recurrence are: large polyp size $(\geq 40 \mathrm{~mm})$, intraprocedural bleeding, and high-grade dysplasia [9]. Among the four factors, piecemeal resection and intraprocedural bleeding are directly related to the procedure itself. Thus, a better endoscopic resection technique should have a higher successful en bloc resection rate and a lower intraprocedural bleeding rate than standard EMR.

Underwater EMR (UEMR) was proposed as an alternative to standard EMR in 2012 [10]. The procedure is done in a decompressed colonic segment. Water is infused into the polyp-bearing segment, which allows the endoscopist to continuously see the polyp. In contrast to standard EMR, UEMR does not require a submucosal injection because the buoyancy effect on the adenoma-bearing mucosa raises it from the deeper muscularis propria. During the past 6 years, about 30 studies on UEMR were published, and they indicated the usefulness of UEMR for large sessile colon polyps, [11] polyps in difficult locations, [12-14] duodenal non-ampullary adenomas, [15] neuroendocrine tumors, $[16,17]$ and recurrent colorectal polyps [18]. However, only three reports compared clinically relevant outcomes of colorectal UEMR and standard EMR. A retrospective cross-sectional study [18] showed better endoscopic complete resection rates for salvage UEMR than for standard EMR when treating recurrent adenomas after previous piecemeal EMR. A retrospective cohort study [19] reported that there were no significant differences in the en bloc resection rate and safety of UEMR and EMR for treatment-naïve large ( $\geq 15 \mathrm{~mm}$ ) colorectal polyps. Another retrospective cohort study [20] reported shorter resection time for UEMR compared with EMR. All of the studies are unmatched studies which are prone to biased confounders. Since no well-designed comparative study was published, the actual benefits of UEMR over standard EMR are unclear.

In this study, we compared the effectiveness and safety of UEMR and standard EMR in patients with colorectal sessile polyps in a large retrospective cohort (>170 polyps per group) using propensity score (PS) matched analysis.

\section{Patients and methods}

\section{Study population and study design}

In this retrospective observational study, we reviewed the prospectively collected electronic medical records and endoscopic images and videos of patients who had consecutively undergone EMR or UEMR of sessile colon polyps in the interventional endoscopy clinics at Chimei Medical Center and Chimei Chiali Hospital between August 2012 and November 2017. Inclusion criteria were: age $\geq 20$ years and medium to large lesions $(\geq 10 \mathrm{~mm}$ ) with Paris classification 0 -Is, Ila, or Ilc morphology ( $\vee$ Fig.1). All patients provided signed written informed consent for each procedure. The study was approved by the Chimei Medical Center Institutional Review Board (IRB No.: 10704002).

We hypothesized that UEMR is not inferior to EMR for endoscopic en bloc resection of colorectal polyps. Sample sizes were calculated based on noninferiority testing [21]. Because there was no study comparing en bloc resection rates of EMR and
Patients with sessile polyps received endoscopic

resection from August 2012 to November 2017 ( $n=463$ polyps, from 408 patients)

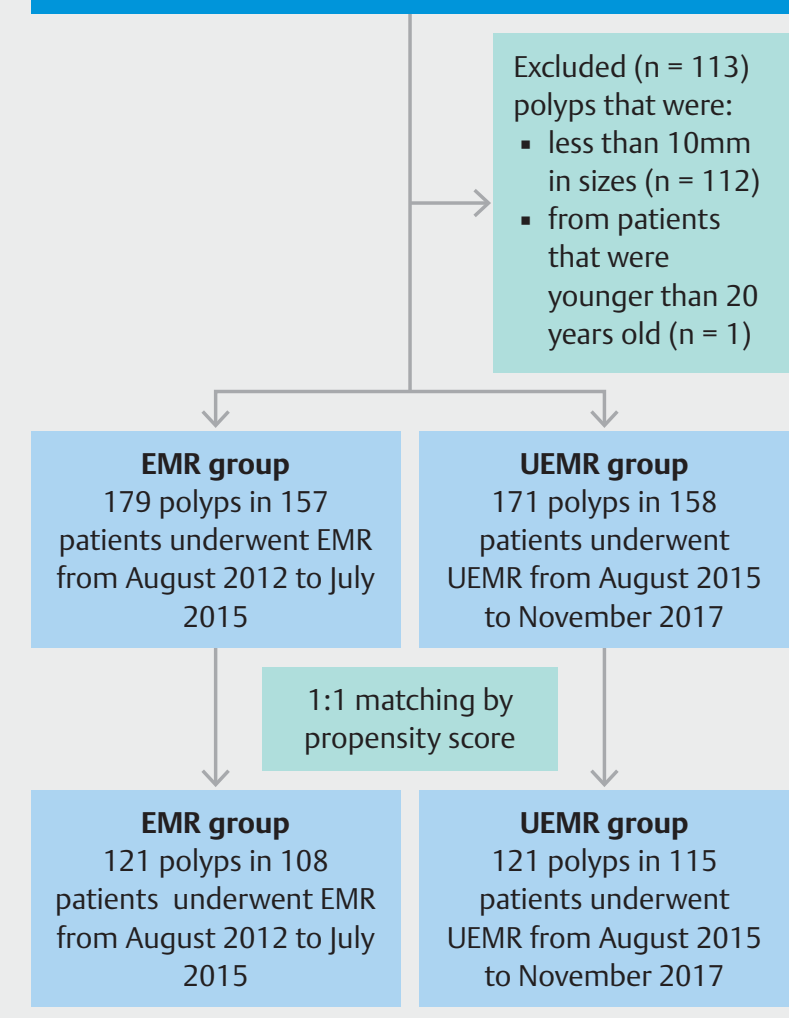

- Fig. 1 Flowchart of patient enrollment.

UEMR by the time we designed this study, we applied the results from observational studies for sample size calculation (en bloc resection rates, EMR: 66\%, [22] UEMR: $81 \%$ [23]). Under the power of 0.8 and $95 \%$ two-sided confidence interval, 75 cases in each group would be required to achieve the desired power to examine the non-inferiority margin of $5 \%$.

\section{Procedures}

Colonoscopies were performed by one experienced endoscopist who had performed more than 200 EMRs before this study. All patients underwent standard bowel cleansing with 2 $\mathrm{L}$ of polyethylene glycol (same-day or 2-day preparation). The bowel preparation scale in the polyp-bearing segment was documented using Aronchick scale [24]. Patients on antiplatelet agents or anticoagulants were asked to keep or discontinue their medications according to the most up-to-date American Society of Gastrointestinal Endoscopy (ASGE) guidelines [25, 26]. An intramuscular or intravenous injection of hyoscine- $\mathrm{N}$ butylbromide $(20 \mathrm{mg}$ ) was routinely given 3 minutes before colonoscopy to prevent bowel spasms. Conscious sedation with propofol was given by an anesthetist based on patient preference. A standard-resolution or high-resolution colonoscope with or without an auxiliary water jet (CF-Q260AL, CF-H290 L, or PCF-Q260AZI: Olympus Medical Systems, Co. Ltd., Tokyo, Ja- 
pan) was used. A transparent hood (D-201-13404: Olympus) was attached to the distal end of the colonoscope to facilitate endoscopic insertion, observation, and resection. Polyp size was estimated by comparison with the width of the transparent hood $(13.4 \mathrm{~mm})$. The morphology of each polyp was described based on the Paris classification [27,28]. The location of each polyp was determined based on the anatomic features of each colon segment. Right-side colon was defined as colon segments proximal to splenic flexure. Left -ide colon was defined as colon segments distal to splenic flexure.

All patients who met the inclusion criteria from August 2012 through July 2015 received conventional EMR, which was done using an oval electrosurgical snare (SD-12U-1, SD-230U-20, or SD-210U-25: Olympus) after the polyp had been submucosally injected with $0.9 \%$ saline solution. The electrosurgical generator's (ESG-100: Olympus) cautery setting was Pulsecut Slow 60 W.

All patients who met the inclusion criteria from August 2015 through November 2017 received UEMR, which was done using a standardized method. Sterile distilled water at room temperature was infused into the polyp-bearing segment of the colon with water jet pump, air/water nozzle, or through the working channel until adequate luminal water filling made the polyp visible. All bubbles in the operative field were suctioned. We then opened a snare around the polyp and resected it using the same cautery setting to the EMR.

In both groups, en bloc resection of all polyps was attempted whenever possible. Piecemeal resection was done when en bloc resection failed or was impossible. Any mucosa suspected of harboring a residual adenoma was removed using a snare or biopsy forceps and then separately sent for a pathology examination. The residual mucosa that could not be grasped and removed using a snare was ablated using snare-tip coagulation with the electrosurgical generator's cautery setting at Soft Coagulation $60 \mathrm{~W}$. If intraprocedural bleeding occurred, it was treated by electrocoagulation with snare tip (Soft Coagulation $100 \mathrm{~W}$ ) or by hemoclips. Postoperative mucosal defects were routinely closed using hemoclips (HX-610-090L: Olympus) if the polyp was $>15 \mathrm{~mm}$.

Follow-up was done in an outpatient clinic visit at 7 to 14 days after the procedure to monitor bleeding, perforation, and any other adverse events.

\section{Variables}

The variables in this study include age $(<50,50-64,65-79$, or $>80$ years); sex (male or female); colon preparation (good-excellent or fair); usage of water jet colonoscope (yes or no); tumor location (right-side or left-side colon); tumor size (10-19, $20-24,25-29$, or $>30 \mathrm{~mm}$ ); tumor morphology (type 0 -Is, type 0 -Ila, granular type lateral spreading tumor (LST-G); nongranular type lateral spreading tumor (LST-NG), or sessile serrated lesions (SSA/P); tumor pathology (adenoma without high-grade dysplasia, adenoma with high-grade dysplasia or carcinoma in situ, T1-shallow $(<1 \mathrm{~mm}$ beyond the muscularis mucosae), T1-deep ( $\geq 1 \mathrm{~mm}$ beyond the muscularis mucosae), or SSA/P); and prior endoscopic manipulation including biopsy or resection on the target lesion (yes or no).

\section{Outcome measurements}

The primary endpoint was the rate of endoscopic en bloc resection. En bloc resection was defined as one-piece resection without any visible residual tissue on conventional white light imaging, narrow band imaging (NBI), or indigo carmine chromoendoscopy. The algorithm to define an endoscopic en bloc resection is shown in Supplemental Fig. 1.

Procedure time was the secondary endpoint for effectiveness. Procedure time for EMR was measured from insertion of the injection needle into the working channel until completion of the treatment, which included time needed for polyp resection, management of bleeding, and closure of the mucosal defect by hemoclips. Procedure time for UEMR was measured from infusing water into the polyp-bearing bowel segment until completion of the treatment.

Safety endpoints included: 1) intraprocedural bleeding that required endoscopic hemostasis; 2) delayed bleeding within 14 days after resection; 3 ) visible muscle defect during resection; and 4) delayed perforation. A muscle defect was defined as visual evidence of partially or completely interrupted muscle fibers on the wound base during resection. Delayed perforation was defined as pneumoperitoneum or ascites with or without peritoneal signs that occurred within 14 days after resection.

\section{Histological evaluation}

All resected specimens were sent to histological examinations to check the pathology, and, for cancerous polyps, the depth of invasion and resection margins. The pathologists serially cut the specimens at 3-mm intervals and determined whether they had any cancerous components. If a specimen was cancerous (T1 or carcinoma in situ), the fixed block was further cut at 1to 2-mm intervals to determine whether there was any deeper invasion or lymphovascular invasion. Depth of invasion in a cancerous polyp was documented as T1-shallow $(<1 \mathrm{~mm}$ beyond the muscularis mucosae) or T1-deep ( $\geq 1 \mathrm{~mm}$ beyond the muscularis mucosae). The status of the lateral and vertical resection margin in a cancerous polyp was also documented.

\section{Propensity score model}

To adjust for potential confounders, we matched each UEMR polyp with one EMR polyp with the propensity score (PS). We used a multivariate logistic regression model to calculate the PS for undergoing UEMR, and performed 1:1 greedy matching algorithm within the caliper width at 0.2 standard deviation (SD) of the PS [29]. Variables in the PS model included age; sex; colon preparation; polyp location, size, morphology, histology, usage of water jet; and prior endoscopic manipulation of the target polyp. As current understanding for the confounders of EMR versus UEMR on treatment outcomes has been limited, we included factors that were reported to be associated with en bloc resection and bleeding rates in the literature in the propensity score model $[6,7,22,30]$. We evaluated performance of our PS model and the matching algorithm by examining the balance across all baseline covariates between the groups after matching using standardized differences (d). A d-value $>0.1$ indicated a significant difference between groups [31]. 


\section{Subgroup and sensitivity analyses}

Subgroup analyses were performed for middle-sized (10$19 \mathrm{~mm}$ ) and larger ( $\geq 20 \mathrm{~mm}$ ) polyps. We grouped polyps by size, matched them with PS, and reported effectiveness and safety outcomes in both groups.

As multi-polyps are not unusual and polyps in the same individual were considered as correlated or clustered data, we conducted a sensitivity analysis by including the first polyp treated for each patient to justify whether our result was affected by correlated data contributed by patients with multiple polyps.

\section{Analytical approach}

We collected and analyzed the data with a polyp as the unit; i.e., two polyps in the same patient were dealt with as two independent polyps in the analysis. However, polyps from the same patient could be correlated by sharing common characteristics, bleeding tendency, adequacy of colon preparation, and underlying diseases.

Continuous variables were shown as mean an SD, and categorical variables as percentages to describe the characteristics of EMR and UEMR patients. To show the rate of differences in en bloc resection rate and bleeding and muscle defects in the EMR and UEMR groups, we presented estimates for the rate differences and their $95 \%$ confidence intervals (95\% Cls). To compare procedure times between UEMR and EMR, we showed the estimated time differences and the $95 \%$ Cls. SAS 9.4 (SAS Institute Inc) was used for all analysis.

\section{Results}

During the study period, 408 patients received standard EMR or UEMR. We excluded one polyp from one patient that was younger than 20 years old. Also excluded were 112 smaller polyps (<10 mm) from 92 patients, leaving 350 polyps (mean size: $16.9 \mathrm{~mm}$; mean procedure time: $10.0 \mathrm{~min}$ ) in 315 patients (mean age: 64.4 years old) as study subjects. From August 2012 through July 2015, standard EMR was used to treat 179 polyps in 157 patients, and from August 2015 through November 2017, UEMR was used to treat 171 polyps in 158 patients. A flowchart of patient enrollment is shown in > Fig. 1.

In the full cohort, the UEMR group was older; had better coIon preparation, had larger polyps, and had procedures done with water-jet colonoscopes more often ( $\downarrow$ Table 1$)$. Nearly all baseline characteristics became balanced (standardized differences $<0.1$ between EMR and UEMR) after PS matching. The distribution of PS score in both groups before and after the matching is shown in > Fig. 2.

In the full cohort, en bloc resection rates in the UEMR groups were not inferior to EMR (EMR: $86.0 \%$ vs. UEMR: $82.5 \%$, rate difference: $-3.6 \%, 95 \% \mathrm{Cl}:-11.2$ to $4.1 \%$ ) ( Table 2 ), nor was mean procedure time (EMR: $10.2 \mathrm{~min}$ vs. UEMR: $9.7 \mathrm{~min}$, difference: $-0.5 \mathrm{~min}, 95 \% \mathrm{Cl}:-2.1$ to $1.1 \mathrm{~min})$. Significantly more patients in the EMR group had intraprocedural bleeding (EMR: $17.9 \%$ vs. UEMR: $5.3 \%$, rate difference: $-12.6 \%$, $95 \% \mathrm{Cl}:-19.2$ to -6.1$] \%)$. Two EMR patients and three UEMR patients had intraprocedural injuries to the muscle layer of their colon wall, but they were successfully treated using endoscopic clipping. One patient with a history of end-stage renal disease and type 2 diabetes mellitus had a delayed perforation 24 hours after UEMR and required emergent surgery. There was no treatment-related mortality in either group.

In the PS matched cohort, the en bloc resection rate in the UEMR group was not inferior to that in the standard EMR group (EMR: $82.6 \%$ vs UEMR: $87.6 \%$, rate difference: $5.0 \%$, $95 \% \mathrm{Cl}:-4.0$ to $13.9 \%)$ with a shorter mean procedure time (EMR: $10.8 \mathrm{~min}$ vs. UEMR: $8.6 \mathrm{~min}$, difference: $-2.2 \mathrm{~min}, 95 \%$ $\mathrm{Cl}:-4.1$ to $-0.3 \mathrm{~min})$. The intraprocedural bleeding rate was significantly lower in the UEMR group (EMR: $15.7 \%$ vs. UEMR: $5.8 \%$, rate difference: $-9.9 \%, 95 \% \mathrm{Cl}:-17.6$ to $-2.2 \%)$. Rates of delayed bleeding and colon wall injury were low in both groups ( $\triangleright$ Table 2 ).

In the subgroup analyses of medium-sized polyps (10 to $19 \mathrm{~mm})$, the en bloc resection rate, intraprocedural bleeding rate, and procedure times between EMR and UEMR were not significantly different ( $\triangleright$ Table 3 ). However, for large polyps $(\geq 20 \mathrm{~mm})$, our results indicated that UEMR was not inferior to EMR in en bloc resection rate (UEMR: $69.1 \%$ vs. EMR: $52.4 \%$, rate difference: $16.7 \%, 95 \% \mathrm{Cl}:-3.9$ to $37.7 \%$ ) but with better safety profile in intraprocedural bleeding. Intraprocedural bleeding was decreased by around one- quarter in the UEMR group (UEMR: $4.8 \%$ vs. EMR: $28.6 \%$, rate difference: $-23.8 \%$, $95 \% \mathrm{Cl}:-38.9$ to $-8.7 \%)$. The procedure time was 5 minutes shorter (UEMR: $12.0 \mathrm{~min}$ vs. EMR: $17.7 \mathrm{~min}$, time difference: $-5.2 \mathrm{~min}, 95 \% \mathrm{Cl}:-8.9$ to $-1.6 \mathrm{~min})$ in the UEMR group ( $\mathrm{T}$ Table 3 ).

Results of sensitivity analysis were similar to those of the primary analysis (Supplementary Table 1 and Supplementary Table 2). Among the PS-matched cohort of the sensitivity analysis, the point estimate of the en bloc resection rates was (EMR: $83.3 \%$ vs. UEMR: $87.7 \%$, rate difference: $4.4 \%, 95 \% \mathrm{Cl}:-4.7$ to $13.5 \%)$. Mean procedure time was shorter in the UEMR group (UEMR: $8.4 \mathrm{~min}$, EMR: $11.1 \mathrm{~min}$; time difference: $-2.7 \mathrm{~min}$, $95 \% \mathrm{Cl}:-4.6$ to $-0.7 \mathrm{~min})$, and the rate of intraprocedural bleeding was lower in the UEMR group than that of EMR group (EMR: $18.4 \%$, UEMR: $3.5 \%$, rate difference: $-14.9 \%$, $95 \% \mathrm{Cl}:-22.8$ to $-7.0 \%)$.

\section{Discussion}

UEMR has shown its potential benefit for resecting sessile colorectal polyps in several case series and cohort studies. In the pioneering study [10], 62 sessile polyps (mean size: $33 \mathrm{~mm}$ ) were piecemeal resected using UEMR. There was only one recurrence (mean follow-up: 20.4 weeks). The safety profile was also acceptable with a delayed bleeding rate of $5 \%$ and perforation rate of $0 \%$. In a follow-up study [32] on 53 LSTs (median size: $30 \mathrm{~mm}$ ) that attempted en bloc UEMR with a large snare, the success rate was $55 \%$, and the recurrence rate was $5 \%$ (mean follow-up: 31 weeks). There was only one instance of delayed bleeding and there were no perforations. These two studies suggest that UEMR is safe and efficacious.

Despite UEMR's being a novel technique, it is considered to be a minor variant of EMR; thus, endoscopists who are familiar 
Table 1 Patient and polyp characteristics.

\begin{tabular}{|c|c|c|c|c|c|c|c|c|}
\hline & \multicolumn{4}{|c|}{ Original cohort } & \multicolumn{4}{|c|}{ Propensity score matched cohort ${ }^{1}$} \\
\hline & EMR & UEMR & d & $P$ & EMR & UEMR & d & $P$ \\
\hline No. of polyps & 179 & 171 & & & 121 & 121 & & \\
\hline Age (mean, SD) & $63.4(9.9)$ & $65.4(11.7)$ & .18 & .03 & $64.2(10.0)$ & $64.1(12.3)$ & .00 & .95 \\
\hline Sex & & & .01 & .91 & & & .05 & .73 \\
\hline Male & $112(62.6)$ & $108(63.2)$ & & & $76(62.8)$ & $79(65.3)$ & & \\
\hline Female & $67(37.4)$ & $63(36.8)$ & & & $45(37.2)$ & $42(34.7)$ & & \\
\hline Colon preparation & & & -.23 & .03 & & & .00 & 1.00 \\
\hline Good-excellent & $163(91.1)$ & $165(96.5)$ & & & $115(95)$ & $115(95)$ & & \\
\hline Fair & $16(8.9)$ & $6(3.5)$ & & & $6(5)$ & $6(5)$ & & \\
\hline Polyp location & & & -.01 & .87 & & & -.08 & 1.00 \\
\hline Left colon & $79(46.2)$ & $77(43)$ & & & $51(42.1)$ & $56(46.3)$ & & \\
\hline Right colon & $100(58.5)$ & $94(52.5)$ & & & $70(57.9)$ & $65(53.7)$ & & \\
\hline Polyp size & & & .32 & $<.01$ & & & .04 & .70 \\
\hline Mean (SD) & $15.8(6.0)$ & $18.0(7.6)$ & & & $16.6(6.5)$ & $17.0(7.2)$ & & \\
\hline Group & & & .32 & $<.01$ & & & .06 & .97 \\
\hline $10-19 \mathrm{~mm}$ & $128(71.5)$ & $98(57.3)$ & & & $79(65.3)$ & $78(64.5)$ & & \\
\hline $20-24 \mathrm{~mm}$ & $35(19.6)$ & $43(25.1)$ & & & $28(23.1)$ & $27(22.3)$ & & \\
\hline $25-29 \mathrm{~mm}$ & $9(5)$ & $17(9.9)$ & & & $8(6.6)$ & $10(8.3)$ & & \\
\hline$>30 \mathrm{~mm}$ & $7(3.9)$ & $13(7.6)$ & & & $6(5)$ & $6(5)$ & & \\
\hline Polyp morphology & & & .23 & .21 & & & .14 & .75 \\
\hline Type 0 -Is & $76(42.5)$ & $54(31.6)$ & & & $42(34.7)$ & $49(40.5)$ & & \\
\hline Type Ila (LST-G) & $31(17.3)$ & $37(21.6)$ & & & $22(18.2)$ & $20(16.5)$ & & \\
\hline Type Ila (LST-NG) & $50(27.9)$ & $56(32.7)$ & & & 41 (33.9) & $40(33.1)$ & & \\
\hline SSA/P & $22(12.3)$ & $24(14)$ & & & $16(13.2)$ & $12(9.9)$ & & \\
\hline Prior manipulation & & & .15 & .15 & & & .07 & .58 \\
\hline None & $132(73.7)$ & $114(66.7)$ & & & $84(69.4)$ & $80(66.1)$ & & \\
\hline Yes & $47(26.3)$ & $57(33.3)$ & & & $37(30.6)$ & $41(33.9)$ & & \\
\hline Polyp pathology & & & .22 & .37 & & & .14 & .89 \\
\hline Adenoma without HGD & $84(46.9)$ & $77(45)$ & & & $57(47.1)$ & $59(48.8)$ & & \\
\hline HGD or CIS & $65(36.3)$ & $63(36.8)$ & & & $45(37.2)$ & $45(37.2)$ & & \\
\hline Adenocarcinoma (T1-shallow) & $1(0.6)$ & $6(3.5)$ & & & $1(0.8)$ & $2(1.7)$ & & \\
\hline Adenocarcinoma (T1-deep) & $4(2.2)$ & $5(2.9)$ & & & $2(1.7)$ & $3(2.5)$ & & \\
\hline SSA/P & $25(14)$ & $20(11.7)$ & & & $16(13.2)$ & $12(9.9)$ & & \\
\hline Water jet scope & & & .55 & $<.01$ & & & .00 & 1.00 \\
\hline No & $141(78.8)$ & $92(53.8)$ & & & $86(71.1)$ & $86(71.1)$ & & \\
\hline Yes & $38(21.2)$ & $79(46.2)$ & & & $35(28.9)$ & $35(28.9)$ & & \\
\hline
\end{tabular}




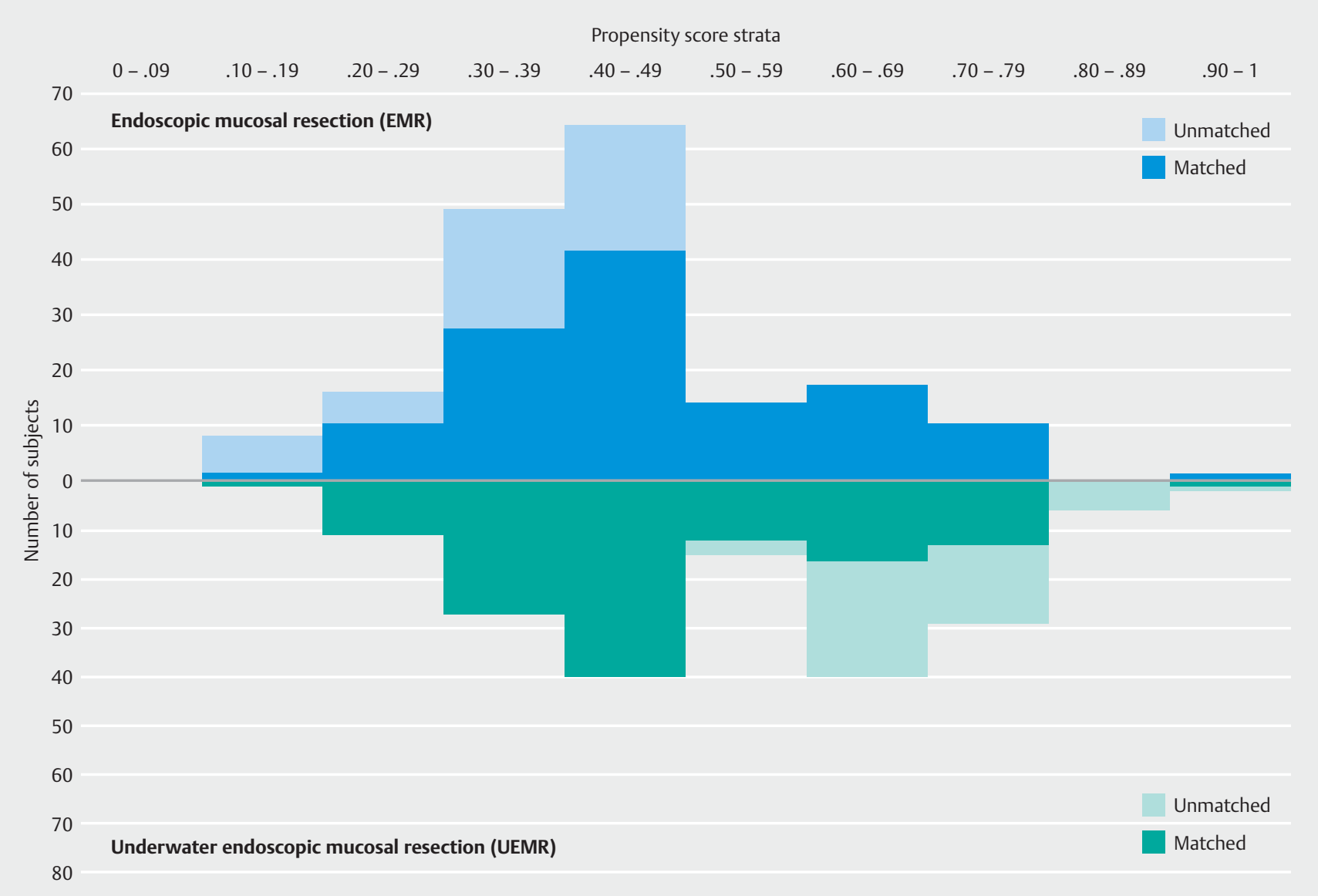

- Fig. 2 Distribution of propensity scores in the EMR and UEMR groups.

- Table 2 Comparison of resection outcomes.

\begin{tabular}{|c|c|c|c|c|c|c|}
\hline & \multicolumn{3}{|c|}{ Original cohort } & \multicolumn{3}{|c|}{ Propensity score matched cohort } \\
\hline & EMR & UEMR & Difference (95\% Cl) & EMR & UEMR & Difference $(95 \% \mathrm{Cl})$ \\
\hline No. of polyps & 179 & 171 & & 121 & 121 & \\
\hline \multicolumn{7}{|l|}{ Resection result } \\
\hline En bloc & $154(86)$ & $141(82.5)$ & $-3.6([-11.2]-4.1)$ & $100(82.6)$ & $106(87.6)$ & $5.0([-4.0]-13.9)$ \\
\hline Piecemeal & $25(14)$ & $30(17.5)$ & & $21(17.4)$ & $15(12.4)$ & \\
\hline \multicolumn{7}{|l|}{ Resection time } \\
\hline Mean (SD) & $10.2(7.9)$ & $9.7(7.7)$ & $-0.5([-2.1]-1.1)$ & $10.8(8.3)$ & $8.6(6.4)$ & $-2.2([-4.1]-[-0.3])$ \\
\hline \multicolumn{7}{|l|}{ Bleeding } \\
\hline Intraprocedural & $32(17.9)$ & $9(5.3)$ & $-12.6([-19.2]-[-6.1])$ & $19(15.7)$ & $7(5.8)$ & $-9.9([-17.6]-[-2.2])$ \\
\hline Delayed & $2(1.1)$ & $2(1.2)$ & $0.1([-2.2]-2.3)$ & $1(0.8)$ & $1(0.8)$ & $0.0([-2.3]-2.3)$ \\
\hline \multicolumn{7}{|l|}{ Colon wall injury } \\
\hline Muscle defect & $2(1.1)$ & $3(1.8)$ & $0.6([-1.9]-3.1)$ & $2(1.7)$ & $1(0.8)$ & $-0.8([-3.6]-2.0)$ \\
\hline Delayed perforation & $0(0)$ & $1(0.6)$ & $0.6([-0.6]-1.7)$ & $0(0)$ & $1(0.8)$ & $0.8(-0.8-2.4)$ \\
\hline
\end{tabular}

EMR, standard endoscopic mucosal resection; UEMR, underwater endoscopic mucosal resection; d, standardized difference; SD, standard deviation

with standard EMR must quickly master UEMR technique. Three recent Western studies [11, 33,34] confirmed that endos- 
- Table 3 Comparison of Resection Outcomes, stratified by sizes of polyps and matched by propensity score

\begin{tabular}{|c|c|c|c|c|c|c|}
\hline & \multicolumn{2}{|c|}{ Polyps $10-19 \mathrm{~mm}^{1}$} & \multirow[t]{2}{*}{ Difference $(95 \% \mathrm{Cl})$} & \multicolumn{2}{|c|}{ Polyps $\geq 20 \mathrm{~mm}^{1}$} & \multirow[t]{2}{*}{ Difference $(95 \% \mathrm{Cl})$} \\
\hline & EMR & UEMR & & EMR & UEMR & \\
\hline Sample size, number & 74 & 74 & & 42 & 42 & \\
\hline En bloc resection & $71(96.0)$ & $72(97.3)$ & $1.4([-4.5]-7.2)$ & $22(52.4)$ & $29(69.1)$ & $16.7([-3.9]-37.3)$ \\
\hline Mean resection time (SD) & $7.1(5.4)$ & $6.1(3.3)$ & $-1.0([-2.5]-0.4)$ & $17.7(9.3)$ & $12(7.4)$ & $-5.2([-8.9]-[-1.6])$ \\
\hline Intraprocedural bleeding & $6(8.1)$ & $3(4.1)$ & $-4.1([-11.7]-3.6)$ & $12(28.6)$ & $2(4.8)$ & $-23.8([-38.9]-[-8.7])$ \\
\hline
\end{tabular}

copists skilled in standard EMR easily adapted to UEMR without specific dedicated training and succeeded with en bloc resection of more than half of their target polyps with almost no adverse events.

\section{UEMR as an alternative to standard EMR}

To be considered as an alternative, the effectiveness and safety of UEMR must be similar or better than conventional EMR. In this study, we evaluated the effectiveness of UEMR based on rates of en bloc resection and the safety of UEMR based on rates of intraprocedural bleeding and adverse events.

We found that the overall en bloc resection rates for UEMR were not inferior to EMR. Moreover, the intraprocedural bleeding rate was lower and the procedure time was shorter in the UEMR group. Our study included the largest number of subjects of any published study comparing the effectiveness and safety of UEMR with conventional EMR. It is also the only study that uses propensity score matching to eliminate the effect of potential confounders.

\section{Effectiveness of UEMR}

Several parameters such as en bloc resection rate, complete resection rate, and recurrence rate measure the effectiveness of an endoscopic resection technique. We chose endoscopic en bloc resection rate as our primary endpoint for three reasons. First, en bloc resection allows a detailed histologic examination of resected specimens [35]. According to National Comprehensive Cancer Network (NCCN) guidelines, a curative resection for a malignant polyp should be considered only if it can be resected en bloc and if the pathologic features are favorable. Any piecemeal resection of a malignant polyp must be considered to be noncurative [36]. Second, en bloc resection is a good surrogate marker because polyp recurrence after en bloc resection is as low as $3 \%$ [8]. Third, it can be evaluated immediately during the procedure by endoscopic appearance.

For polyps $<20 \mathrm{~mm}$, standard EMR was the default treatment in Japanese, American, and European guidelines [3-5] because of its ease of use and effectiveness [37]. We found that en bloc resection rates in polyps $<20 \mathrm{~mm}$ were $>96.0 \%$ for EMR and $97.3 \%$ for UEMR, and we confirmed the efficacy of UEMR for polyps $<20 \mathrm{~mm}$. UEMR could be an alternative to standard EMR in this size group.
For polyps $\geq 20 \mathrm{~mm}$, there are still debates about which should be the default technique. In Japanese and European guidelines, standard EMR is believed to lead to piecemeal resection, thus they recommend not using it for LST-NGs $>20 \mathrm{~mm}$ or for polyps suspected of being malignant. In American guidelines, piecemeal EMR is considered acceptable if complete resection of the polyp is anticipated and if there is no invasive cancer in the resected tissue. The en bloc resection rate with standard EMR for polyps $\geq 20 \mathrm{~mm}$ ranged from $12 \%$ to $66 \%$ [22], and that of UEMR ranged from $20 \%$ to $68 \%[11,19,20,32,34,38]$. Our UEMR en bloc resection rate for polyps $\geq 20 \mathrm{~mm}(69.1 \%)$ was also not inferior to standard EMR (52.4\%). Thus UEMR could also be an alternative to standard EMR in this size group. In our experience, submucosal injection expanded the width of polyp base but the underwater technique won't. Thus the width of the polyp base may look smaller and easier to be entrapped by snare. The phenomenon seems to be more significant in larger than in smaller lesions. It is our assumption that is why the en bloc resection rate looks higher for larger polyps in UEMR group.

When comparing UEMR with ESD, despite having better safety profiles, the en bloc resection rate of UEMR for resecting polyp $\geq 20 \mathrm{~mm}$ is not as good as ESD, which was reported to be more than $90 \%$ [22].

\section{Intraprocedural bleeding and procedure time}

The intraprocedural bleeding rate in our UEMR group (overall: $5.8 \%$, polyps $\geq 20 \mathrm{~mm}: 4.8 \%$ ) was much lower than previous EMR literature $(13 \%-26.5 \%)$ [30], while the intraprocedural bleeding rate in our standard EMR group (overall: $15.7 \%$ ) was similar to previous literature. Accordingly, time required for hemostasis was less in UEMR group and it contributed to reduction in overall procedure time. Although most intraprocedural bleeding can be managed using endoscopic hemostasis and is not considered as a complication, it usually interrupts and prolongs the resection procedure. Moreover, it has also been reported to be an important risk factor associated with local recurrence in EMR piecemeal resection [9,30]. In our experience, the submucosa in the ulcer base remained thick and submucosal vessels stayed intact after UEMR, while the submucosal vessels often cut at standard EMR. However, this is only our subjective assumption. To our knowledge, there is no scientific evi- 
dence regarding this finding. The actual reason for UEMR causes less intraprocedural bleeding requires further investigation. Regarding the procedure time, as observed in previous study [20], the mean procedure time of UEMR is also less than standard EMR (overall: 2.2 mins less, for polyps $\geq 20$ mm: 5.7 mins less). In these regards, UEMR is advantageous over standard EMR.

\section{Adverse events}

Risks of delayed bleeding, muscle layer injury, and perforation were not significantly different between UEMR and EMR in the current study. In standard EMR, submucosal injection helps prevent these complications by creating a cushion between the polyp and the muscularis layer to prevent deep thermal injury and accidental ensnaring of the deep muscularis propria. In UEMR, it is hypothesized that water immersion in a nondistended colon "floats" the mucosa and submucosal away from the deeper muscularis layer, thus mimicking the "cushion" effect of submucosal injection in standard EMR. There is only one case of UEMR-related perforation among the more than 400 UEMR cases in the published studies [39]. In our study results, we found that UEMR didn't eliminate the risk of muscle-layer injury. Care should be still taken when snaring polyps.

We also had one case of delayed perforation after UEMR. No delayed perforation after UEMR has been previously reported. The patient in this case was a 73-year-old woman who was undergoing regular hemodialysis for her end-stage renal disease. She had a 13-mm laterally spreading tumor non-granular type in her descending colon. The polyp was resected in one piece without a muscle defect. However, we used the snare tip to thermocoagulate an exposed oozing vessel on the wound base. Delayed perforation occurred 24 hours after UEMR. An 8$\mathrm{mm}$ perforation was seen in her descending colon during an explorative laparotomy. She recovered after a colectomy. We suspected that the delayed perforation was related to the post-resection thermocoagulation rather than to the UEMR procedure itself.

\section{Strengths and limitations}

The basic strength of our study is the large sample size in a realworld setting. We enrolled more than 170 polyps in each arm and matched 121 pairs for analysis. The sample size is more than any single UEMR study $[10-14,18,19,32,34,38-40]$. Besides, this is the only comparative effectiveness study that applied sophisticated PS matching approaches to balance numerous confounding factors that may influence the outcome estimates.

Our study has some limitations. First, this is a study of singlecenter and single-operator design and it is unclear whether the results could be extrapolated to other institutions with multiple operators. However, internally validity must be established before one can consider whether the results are externally valid. As we applied a PS model to ensure the comparability between groups and the findings were robust in sensitivity analysis, our results are valid and provide evidence of the comparativeness effectiveness/safety outcome of EMR and UEMR in the realworld setting. Second, given that UEMR was performed in re- cent cases, improvements in EMR techniques over time might contribute to a better safety profile in UEMR cases. However, given that the learning curve for EMR often plateaus at 100 cases [41] and the operator had performed more than 200 cases of EMR before recruiting patients for this study, the learning effect of EMR techniques could only have minimal effects on our results. Third, because we only had limited experience in UEMR before August 2015 when UEMR was introduced to our institute, the superiority of UEMR over EMR could be underestimated. However, we considered the impact to be minimal because the previous report showed that UEMR was easily learned by endoscopists already skilled in EMR [33]. Fourth, resected polyps with endoscopically invisible residual tissue might be misdiagnosed as an en bloc resected polyp and could result in misclassification bias. However, we believed that there were only limited missing cases and the error was considered non-differential and could only drive the estimates toward to null [42]. Finally, due to the relatively short follow-up time, long-term outcomes including local recurrence rates and interval cancers could not be addressed in our study. We need further studies to clarify if UEMR is comparable to conventional EMR in the long-term outcomes.

\section{Conclusion}

Regarding short-term outcomes, UEMR is advantageous compared with standard EMR in resecting colorectal sessile polyps because it yields comparable en bloc resection rate with less intraprocedural bleeding and shorter procedure time. Additional investigations of outcomes, including long-term outcomes, in prospective randomized trials are warranted.

\section{Competing interests}

None

\section{References}

[1] Ferlay JSI, Ervik M, Dikshit R et al. GLOBOCAN 2012 v1.0, Cancer Incidence and Mortality Worldwide: IARC CancerBase No. 11 [Internet]. Lyon, France: International Agency for Research on Cancer; 2013

[2] Zauber AG, Winawer SJ, O’Brien MJ et al. Colonoscopic polypectomy and long-term prevention of colorectal-cancer deaths. N Engl J Med 2012; 366: $687-696$

[3] Fisher DA, Shergill AK, Early DS et al. Role of endoscopy in the staging and management of colorectal cancer. Gastrointest Endosc 2013; 78: $8-12$

[4] Tanaka S, Kashida H, Saito $Y$ et al. JGES guidelines for colorectal endoscopic submucosal dissection/endoscopic mucosal resection. Dig Endoscopy 2015; 27: 417 - 434

[5] Ferlitsch M, Moss A, Hassan C et al. Colorectal polypectomy and endoscopic mucosal resection (EMR): European Society of Gastrointestinal Endoscopy (ESGE) Clinical Guideline. Endoscopy 2017; 49: $270-297$

[6] Ahmad NA, Kochman ML, Long WB et al. Efficacy, safety, and clinical outcomes of endoscopic mucosal resection: a study of 101 cases. Gastrointest Endosc 2002; 55: 390 - 396 
[7] Hwang JH, Konda V, Abu Dayyeh BK et al. Endoscopic mucosal resection. Gastrointest Endosc 2015; 82: 215-226

[8] Belderbos TD, Leenders M, Moons LM et al. Local recurrence after endoscopic mucosal resection of nonpedunculated colorectal lesions: systematic review and meta-analysis. Endoscopy 2014; 46: 388-402

[9] Tate DJ, Desomer L, Klein A et al. Adenoma recurrence after piecemeal colonic EMR is predictable: the Sydney EMR recurrence tool. Gastrointest Endosc 2017; 85: 647 - 656.e646

[10] Binmoeller KF, Weilert F, Shah J et al. "Underwater" EMR without submucosal injection for large sessile colorectal polyps (with video). Gastrointest Endosc 2012; 75: 1086 - 1091

[11] Uedo N, Nemeth A, Johansson GW et al. Underwater endoscopic mucosal resection of large colorectal lesions. Endoscopy 2015; 47: 172 174

[12] Uedo N, Nemeth A, Toth E et al. Underwater endoscopic mucosal resection of a large depressed adenoma in the ileum. Endoscopy 2014; 46: (Suppl. 01): E336 - 337

[13] Binmoeller KF, Hamerski CM, Shah JN et al. Underwater EMR of adenomas of the appendiceal orifice (with video). Gastrointest Endosc 2016; 83: 638-642

[14] Hamada K, Uedo N, Tanishita H. Underwater endoscopic mucosal resection of an intramucosal carcinoma located from the lower rectum to the anal canal. Digest Endosc 2018; 30: 119-120

[15] Yamasaki Y, Uedo N, Takeuchi Y et al. Underwater endoscopic mucosal resection for superficial nonampullary duodenal adenomas. Endoscopy 2018; 50: 154-158

[16] Anderloni A, Murino A, Jovani M et al. Underwater endoscopic mucosal resection of a duodenal neuroendocrine tumor. Gastrointest Endosc 2016; 83: 259-260

[17] Yamashina T, Tumura T, Maruo T et al. Underwater endoscopic mucosal resection: a new endoscopic method for resection of rectal neuroendocrine tumor grade 1 (carcinoid) $\leq 10 \mathrm{~mm}$ in diameter. Endosc Int Open 2018; 6: E111-e114

[18] Kim HG, Thosani N, Banerjee S et al. Underwater endoscopic mucosal resection for recurrences after previous piecemeal resection of colorectal polyps (with video). Gastrointest Endosc 2014; 80: 1094-1102

[19] Schenck RJ, Jahann DA, Patrie JT et al. Underwater endoscopic mucosal resection is associated with fewer recurrences and earlier curative resections compared to conventional endoscopic mucosal resection for large colorectal polyps. Surg Endosc 2017; 31: 4174-4183

[20] Cadoni S. Underwater endoscopic colorectal polyp resection: Feasibility in everyday practice. United Europ Gastroenterol J 2018; 6: 454-462

[21] Walker E, Nowacki AS. Understanding equivalence and noninferiority testing. J Gen Intern Med 2011; 26: 192-196

[22] Nakajima T, Saito Y, Tanaka S et al. Current status of endoscopic resection strategy for large, early colorectal neoplasia in Japan. Surg Endosc 2013; 27: $3262-3270$

[23] Kawamura T, Sakai H, Ogawa T et al. Feasibility of underwater endoscopic mucosal resection for colorectal lesions: a single center study in Japan. Gastroenterol Res 2018; 11: 274-279

[24] Aronchick CA. Bowel preparation scale. Gastrointest Endos 2004; 60: 1037-1038; author reply 1038-1039
[25] Anderson MA, Ben-Menachem T, Gan SI et al. Management of antithrombotic agents for endoscopic procedures. Gastrointest Endosc 2009; 70: 1060 - 1070

[26] Acosta RD, Abraham NS, Chandrasekhara V et al. The management of antithrombotic agents for patients undergoing Gl endoscopy. Gastrointest Endosc 2016; 83: 3-16

[27] [Anonymous] The Paris endoscopic classification of superficial neoplastic lesions: esophagus, stomach, and colon: November 30 to December 1, 2002. Gastrointest Endosc 2003; 58: S3-43

[28] [Anonymous] Update on the paris classification of superficial neoplastic lesions in the digestive tract. Endoscopy 2005; 37: 570-578

[29] Austin PC. Optimal caliper widths for propensity-score matching when estimating differences in means and differences in proportions in observational studies. Pharm Statistics 2011; 10: 150-161

[30] Sidhu M, Tate DJ, Desomer L et al. The size, morphology, site, and access score predicts critical outcomes of endoscopic mucosal resection in the colon. Endoscopy 2018; 50: 684-692

[31] Austin PC. Balance diagnostics for comparing the distribution of baseline covariates between treatment groups in propensity-score matched samples. Statistics Med 2009; 28: $3083-3107$

[32] Binmoeller KF, Hamerski CM, Shah JN et al. Attempted underwater en bloc resection for large $(2-4 \mathrm{~cm})$ colorectal laterally spreading tumors (with video). Gastrointest Endosc 2015; 81: 713-718

[33] Wang AY, Flynn MM, Patrie JT et al. Underwater endoscopic mucosal resection of colorectal neoplasia is easily learned, efficacious, and safe. Surg Endosc 2014; 28: 1348 - 1354

[34] Curcio G, Granata A, Ligresti D et al. Underwater colorectal EMR: remodeling endoscopic mucosal resection. Gastrointest Endosc 2015; 81: $1238-1242$

[35] Oka S, Tanaka S, Saito Y et al. Local recurrence after endoscopic resection for large colorectal neoplasia: a multicenter prospective study in Japan. Am J Gastroenterol 2015; 110: 697 - 707

[36] Benson AB 3rd, Venook AP, Bekaii-Saab T et al. Colon cancer, version 3.2014. J Nat Comp Cancer Network 2014; 12: 1028 - 1059

[37] Repici A, Pellicano R, Strangio G et al. Endoscopic mucosal resection for early colorectal neoplasia: pathologic basis, procedures, and outcomes. Dis Colon Rectum 2009; 52: $1502-1515$

[38] Siau K, Ishaq S, Cadoni S et al. Feasibility and outcomes of underwater endoscopic mucosal resection for $\geq 10 \mathrm{~mm}$ colorectal polyps. Surg Endosc 2017: doi:10.1007/s00464-017-5960-8

[39] Ponugoti PL, Rex DK. Perforation during underwater EMR. Gastrointestinal endoscopy 2016; 84: 543-544

[40] Sandhu DS, Lee Y], Gerke H. Underwater endoscopic mucosal resection (UEMR): an alternative for removal of large sessile colorectal polyps. Minerva gastroenterologica e dietologica 2017: doi:10.23736/s1121-421x.17.02444-8

[41] Bhurwal A, Bartel M], Heckman MG et al. Endoscopic mucosal resection: learning curve for large nonpolypoid colorectal neoplasia. Gastrointest Endosc 2016; 84: 959-968.e957

[42] Grimes DA, Schulz KF. Bias and causal associations in observational research. Lancet (London, England) 2002; 359: 248-252 